\title{
THE PRESENT POSITION OF RADIOTHERAPY IN THE TREATMENT OF MALIGNANT DISEASE OF THE PELVIS
}

\author{
By M. Lederman, M.B., D.M.R.E. \\ Deputy Director, Radiotherapy Department, Royal Cancer Hospital ; Radiotherapist, Chelsea Hospital for Women; \\ Royal National Throat, Nose and Ear Hospital ; Moorfields, Westminster and Central Eye Hospital
}

For the purposes of this paper the term ' radiotherapy' will be used to cover the established methods of treatment by radium and X-rays. Recent advances in nuclear physics and apparatus construction may materially alter radiotherapy as we know it today by placing in the hands of radiotherapists artificial radioactive substances and machines capable of delivering beams of neutrons, electrons and $\mathrm{X}$-rays of energies varying from ten to hundreds of millions of volts. The potentialities of these new weapons are as yet relatively unexplored and time must elapse before their value in the treatment of cancer can be assessed.

The group of malignant neoplasms of most direct interest and concern to the gynaecologist comprises the cancers arising from the uterus, the ovary, the vulva, the vagina and urethra in order of frequency of occurrence.

Radiotherapy is of considerable value as a palliative agent in the treatment of inoperable tumours arising from all these sites and as a preor post-operative measure, it forms a valuable adjunct to the surgical treatment of cancer of the corpus uteri, the ovary and the vulva. A reasonable claim can be made for radiotherapy as a curative agent in the treatment of cancer of the cervix uteri, vagina and urethra and as such it can be employed in preference to surgery for most operable tumours of these sites.

As the grounds upon which these claims are based are still subject to differences of opinion a clear picture of the present position of radiotherapy in the treatment of pelvic malignan? disease can only be obtained by the separate consideration of all the neoplasms concerned.

\section{Cancer of the Uterus}

The customary subdivision of uterine cancer into corpus and cervix cancer is well founded both upon clinical and therapeutic grounds. Whereas cervix cancer has come to be regarded as an important field for radiotherapy, few are bold enough to make comparable claims with regard to corpus cancer.

\section{Cancer of the Cervix Uteri}

The problem of choice of treatment as between surgery and radiotherapy has been settled beyond all reasonable doubt in favour of radiotherapy. Sufficient evidence has now been accumulated to show that for most cases, both operable and inoperable, radiotherapy, i.e. intra-cavitary radium treatment usually combined with external X-ray therapy, is the treatment of choice. The following facts can be advanced in support of this statement :-

I. Results. It is, after all, the results of treatment that really matter and there is no doubt that for operable cases radiotherapy is at least as successful as surgery. For inoperable cases surgery admittedly has nothing to offer and yet even in this group radiotherapy can still achieve a great deal. Table $\mathrm{I}$, which includes a recent summary of radiotherapy results, shows quite clearly that the latter method can achieve results which on the average compare favourably with some of the best surgical results. It can also be seen that the results from selected radiotherapy centres are considerably better.

2. Treatment Mortality. In" skilled hands the operative mortality of the radical abdominal hysterectomy has been reduçed from I 5 per cent. to 5 per cent. in selected cases. The mortality following radium treatment is $I$ to 2 per cent. and, unlike the surgical mortality, is almost entirely avoidable as it is usually due to sepsis and could be eliminated if radium were not used indiscriminately in the treatment of patients with evidence of pelvic or severe local sepsis.

3. Treatment Complications and Sequelae. Apart from the danger of haemorrhage, shock and sepsis, the chief risks of the surgical treatment of carcinoma of the cervix are damage to the rectum, bladder and ureters. In the case of radiotherapy the chief complications are those due to secondary infection and radiation damage to the tissues. The septic complications can be largely avoided by selection of cases and careful technique ; equally 
Table I

Carcinoma of the Cervix Uteri (Richards, 1947)

(Comparison of Results by Surgery and Radiotherapy)

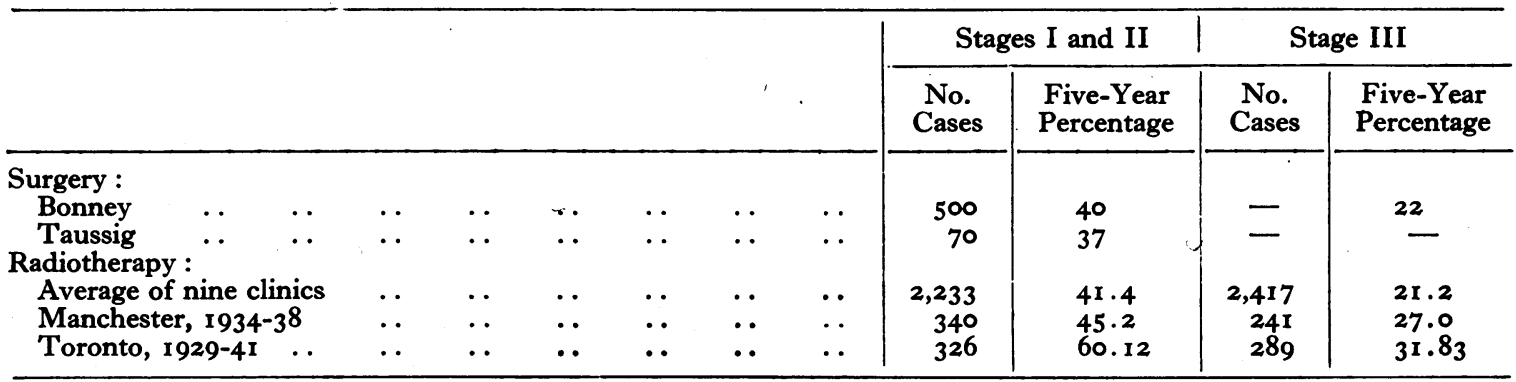

(Reproduced from the 'Proceedings of the Royal Society of Medicine' by permission of the Editors.)

the incidence of the more gross forms of tissue damage such as rectal or bladder fistulae can be reduced to negligible proportions. Late rectal reactions are a distressing and fairly frequent postradiation complication (5 to ro per cent. of cases). They are difficult to avoid if treatment is to be thorough, but there is hope - that improved methods of dose measurement coupled with greater accuracy in correlating radium and X-ray treatment may appreciably lower the incidence of this troublesome complication. Any further enumeration of the many minor or uncommon complications of radiotherapy would serve no more useful purpose than to attempt to cover all the possible risks associated with a surgical procedure.

An important sequel to surgical treatment which does require special comment, since its seriousness is frequently minimized, is the degree of mutilation resulting from operation. The Wertheim operation, if properly performed, virtually involves the extirpation of most of the female genital tract, and it requires little imagination to picture the probable consequences of an operation of this nature on the younger patient's psychological make-up and marital life. By contrast the patient successfully treated by radiotherapy is left with an atrophic, non-functioning but anatomically intact genital tract.

\section{Contraindications to Radiotherapy}

Whilst accepting the fact that most cases of cancer of the cervix are best treated by radiotherapy, it must also be emphasized that there are cases for whose treatment surgery is to be preferred.

The following contraindications to radiotherapy have, therefore, to be borne in mind as they form the chief indications for surgery :-

I. When local conditions exist which prevent the proper application of radium, e.g. the presence of some congenital or acquired anatomical abnormality of the genital tract.

2. For cases complicated by adnexal or pelvic infection. In the absence of constitutional symptoms, local tumour infection or even a pyometra need not seriously interfere with or unduly delay radiation treatment. By contrast the presence of an associated inflammation of the adnexae or pelvic cellular tissues, particularly if chronic or subacute, presents a formidable risk to intra-cavitary treatment. Where surgery is practicable, especially in the chronic or latent cases of this type, it is undoubtedly to be preferred.

3. When some other pelvic tumour exists which requires surgical intervention. The pelvic tumours most likely to be encountered are uterine fibroids or an ovarian cyst. The presence of fibroids is not a contraindication to radium therapy provided the uterus be less than the size of a four-months' pregnancy and the fibroids be mainly interstitial or subperitoneal in position. The presence of a very large fibroid may result in malposition of the radium sources whilst submucous fibroids sometimes undergo necrosis and sloughing after treatment and the patient may then develop a foul septic discharge with constitutional symptoms. Because of the adverse conditions thus introduced, surgical treatment should always be considered in operable cases of this kind.

The presence of an ovarian cyst, if small, need neither delay nor prevent radium treatment. If a large cyst be present its surgical removal with the carcinoma should be considered because the uterus and the radium containers may be displaced by the cyst, or the cyst may, under the influence of radiation, become bound down to adjacent structures and its subsequent removal made difficult. The possible risks of an ovarian cyst becoming infected and complicating the radiation treatment must also be considered. 
4. For operable cases recurring after previous radiotherapeutic treatment. It should be an absolutely inflexible rule that a full course of radium treatment should never be repeated. A second course is unlikely to succeed where the first failed, and if given, the patient's end is likely to be hastened and made worse by the miseries of radionecrosis. The difficulties encountered when operating on ' radium failures' are well recognized, but in view of the fact that the patient's life is at stake, these difficulties should be accepted by the surgeon as a challenge rather than as a contraindication to surgery.

5. When the patient with operable carcinoma refuses radiation treatment, or in rare cases where on psychological grounds it is deemed undesirable to let the patient know the nature of her disease.

6. For operable cases complicated by pregnancy. Most obstetricians advocate the Wertheim operation for operable cases but radiotherapy is being more widely used even for this group.

There are two special indications for surgical as opposed to radiation treatment which are sometimes given and which require comment :-

I. Adenocarcinoma arising from the endocervix. Although this particular type of cervix cancer is alleged to be less radiosensitive than the commoner squamous tumour, there is no real evidence to show that this is in fact the case; nor has it yet been proved that the results following surgical treatment are better than those obtained by radiotherapy.

2. If during irradiation serial histological or cytological examination shows the tumour to be responding unfavourably to treatment. Much promising work has been done on this special radiobiological problem but there is as yet no reasonably certain method of assessing radiosensitivity by histological or cytological methods and therefore judgment must be reserved until the true value of the methods employed can be properly assessed. In this country Glucksmann and Spear (1945) have investigated the response of cervical carcinomata to radiotherapy by quantitative histological analysis of serial biopsies obtained before and during teatment. From their results they estimate that their methods are 90 per cent. accurate when used for assessing prognosis in a given case. Recently Glucksmann and Way (1948) have attempted to use the cell-counting technique as a basis for selecting patients who would be unsuitable for treatment by radiotherapy and for whom surgery would be the method of choice. Whilst their published results do not give the impression that their methods are particularly successful, their approach is certainly rational and merits further investigation.

In America, Ruth Graham (1947) claims that the vaginal smear method of examination gives 88 per cent. accuracy of prognosis at an early stage in radiation treatment. Should this claim be substantiated by other workers, her method should prove of considerable value since operable cases responding unfavourably to radiation could be offered a surgical alternative immediately.

\section{Surgery and Radiotherapy in Combination}

It is known that both surgery and radiotherapy are each capable of curing cancer of the cervix independently, and it is not, therefore, surprising to find that various combinations of the two methods have been explored in the attempt to improve results.

In many clinics, both in Europe and America, a radical hysterectomy is performed on operable cases almost as a routine after a full course of radium treatment has been given, in the hope of preventing recurrence. Hultberg (1944) has reviewed the arguments in favour of this combination and, from a study of his own cases and others reported in the literature, recommends that radical hysterectomy be performed more frequently after radium treatment in the hope that the risk of recurrence may be thereby reduced. Heyman (1943, 1944), on the other hand, believes that if prophylactic hysterectomy be performed routinely the number of patients lost by the operation will be greater than the number saved. $\mathrm{He}$ is, moreover, of the opinion that improvement in radiotherapeutic technique provides a more promising means of avoiding local recurrence than does routine prophylactic hysterectomy.

In the same way that fear of local recurrence is used as an argument for removing the uterus after radium treatment, the alleged inability of radiation to sterilize pelvic lymph node metastases has been used as a reason for advocating surgical removal of these nodes. Taussig (1943) was perhaps the foremost exponent of iliac lymphadenectomy, recommending a careful and thorough dissection of the pelvic lymph nodes as an adjunct to radiation of the cervix and pelvic tissues, chiefly in Stage II cases. He demonstrated that $2 \mathrm{I}$ per cent. of patients with positive nodes subjected to his operation lived five years or more, but his results, 38 per cent. five-year survivals in a series of 70 Stage II cases, compare very unfavourably with the results obtained in most centres for comparable cases treated by radiotherapy alone. It would seem, therefore, that the precise indications for iliac lymphadenectomy in the treatment of cancer of the cervix uteri have yet to be determined.

\section{Cancer of the Corpus Uteri}

In marked contrast to the cervix, surgery is almost universally accepted as the method of choice 
in treating operable cases of corpus cancer. In view of the small operative risk and the good results obtained by panhysterectomy, few radiotherapists would advocate radiation in preference to surgery for the early operable case. Heyman (194I, 1947) is the chief protagonist of radiotherapy for cancer of the corpus uteri. At the Radiumhemmet he employs radiotherapy as the routine primary method of treatment combined with surgery in cases of failure. Surgical treatment is used as the primary method only in operable cases complicated by large fibroids or an ovarian tumour. He has developed a special method of packing the uterus with radium capsules and although his published results are based on cases both operable and inoperable where the disease is limited to the corpus uteri, these results are a remarkable tribute to his skill and the success of the methods he has elaborated. Whilst it must be accepted that the treatment of corpus cancer is primarily surgical, it must be remembered that radium can be used as a curative agent and should be given full consideration in borderline cases or operable cases where the operative risk is excessive.

For inoperable cases radiotherapy is unequalled as a palliative procedure and gives a small but worthwhile percentage of five-year survivals. A combination of surgery and radiotherapy is indicated for tumours which are histologically poorly differentiated when it is known that the results of surgery are poor. Equally, post-operative $\mathrm{X}$-ray therapy or intra-vaginal radium therapy improves the results of surgery by reducing the incidence of recurrence, particularly in the vagina.

\section{Cancer of the Ovary}

The treatment of malignant disease of the ovaries is primarily surgical. Radiotherapy is practically of no value as a curative agent for the following reasons :-

I. The commonest primary malignant ovarian tumour is the papillary adenocarcinoma which is, unfortunately, among the least radiosensitive of ovarian neoplasms. Apart from the radioresistant papillary adenocarcinoma wherein even palliation is difficult to obtain there are other much less common ovarian tumours which are radiosensitive to a varying degree and in whose treatment radiotherapy is of distinct value as a palliative or supplementary measure. These tumours are solid adenocarcinoma, dysgerminoma, malignant granulosa-cell tumour, chorionepithelioma and malignant teratoma.

2. The commoner forms of ovarian cancer are characterized by a tendency to widespread dissemination throughout the peritoneal cavity. This means that in order to deal with the diease properly, irradiation must be applied not only to the true pelvis but to the whole of the peritoneal cavity from the under surface of the liver to the pelvic floor. Unfortunately there is, as yet, no known method of giving radiation to so large a part of the body in doses sufficient to destroy ovarian cancer without risk of the patient succumbing to the treatment.

Although radiotherapy cannot offer a great deal to the patient suffering from ovarian cancer its help is nevertheless frequently sought in the following circumstances :-

I. An ovarian tumour or cyst is removed and on histological examination a malignant neoplasm is found, although macroscopically at operation nothing was found to suggest the true nature of the tumour.

2. A primary malignant neoplasm is found at operation and removed but the removal may be incomplete, evidence of metastases may be found, or rupture of a cyst with spill may have occurred.

3. An inoperable malignant tumour is found, either clinically or at laparotomy.

4. Post-operative recurrence has taken place.

The radiotherapist's problem will clearly differ in each of these groups of cases :-

I. When a tumour which at operation was thought to be benign proves on histological examination to be malignant, provided that the tumour has been completely removed, there is little point in advising post-operative radiotherapy. There is no evidence to show that irradiation of normal tissues is of any prophylactic value as far as the prevention of recurrence or metastasis is concerned. Post-operative radiation should be reserved for those cases where there is reason to believe that malignant tissue still remains even though in microscopic amounts ; and radiation to be effective must be given full dosage. When removal is considered to be complete the patient should be regularly followed up and radiotherapy given only if recurrence takes place. With two possible exceptions this conservative attitude with regard to the post-operative use of radiotherapy should be adopted whatever the circumstances attending the discovery and removal of an operable malignant ovarian tumour. The dysgerminoma and the chorionepithelioma provide the two exceptions since their high degree of malignancy and their radiosensitivity justify the routine use of radiotherapy as a post-operative measure.

2. In the case of incomplete removal or when the contents of a malignant cyst have been spilled into the peritoneal cavity during removal, postoperative radiation is indicated. The method of choice for the post-operative radiation of ovarian neoplasms is X-ray therapy, supplemented in certain cases by intracavitary radium treatment if 
the uterus be present. In this connection, whilst fully recognizing the value of the traditional operation for ovarian cancer, a plea must nevertheless be made for preservation of the uterus in those cases where it is not directly or obviously invaded by neoplasm. In leaving the uterus the surgeon provides the radiotherapist with a useful 'intrapelvic container' for radium, whereas the routine removal of the uterus restricts the radiotherapist without necessarily helping the patient.

3. In advanced inoperable cases, X-ray therapy should be given as a palliative or placebo treatment, care being taken not to let the treatment upset the patient. As a rule, the presence of marked ascites should be regarded as a contraindication to treatment.

4. With post-operative recurrence, surgical treatment is usually impracticable. Depending on the site and extent of the recurrence, X-ray or radium treatment, or both in combination if possible, should be given.

\section{Cancer of the Vulva}

Cancer of the vulva is a muco-cutaneous cancer, but unlike most tumours of this type it responds unfavourably to radiation treatment. The factors chiefly responsible for the poor results of radiotherapy are :-

I. Cancer of the vulva is a disease of elderly women; atrophy vascular degeneration and fibrotic changes are frequently present in the vulval tissues and tumour sepsis is constant. This combination of factors has the effect of impairing both the response of the tumour to radiation and the reparative powers of the tumour bed.

2. Cancer of the vulva is potentially a disease of the whole vulva. Leukoplakic changes are present in nearly half the cases and multi-centric tumours often occur so that cure of one lesion may be followed by reappearance of another outside the region treated. It is clear, therefore, that some method which can deal with the whole vulva, such as excision or diathermy coagulation, is preferable to the localized methods of radiotherapy.

3. Tumours of this site are technically difficult to treat by radiotherapy. External methods of radiation such as $\mathrm{X}$-ray therapy or teleradium are not very satisfactory, and of the local methods of radium therapy implantation alone permits satisfactory treatment in selected cases.

Within these limitations radiotherapy may still be of considerable use in the treatment of vulval cancer. The frankly inoperable and the operable case unsuitable for surgery on general grounds can both be treated, the former palliatively and the latter curatively with, admittedly, a reduced chance of success. In certain cases there is a field of usefulness for pre- and post-operative radiation whilst for the usual case wherein the primary disease is treated surgically, radiotherapy may have some part to play in the treatment of the inguinal lymph node regions. Where inoperable lymph node metastases are present, radiotherapy is the only treatment method possible. Where operable lymph node metastases are present there is little disagreement that block dissection is indicated. The only difficulty arises in cases where there are no clinically detectable lymph node metastases. With the possible exception of the-elderly patient with a very early lesion, all these cases warrant an active policy as far as the lymph node areas are concerned. An observation or 'wait and see' policy is all too frequently a confession of defeat on the part of the gynaecologist and a death warrant for the patient. Berven (I94I) has shown that using his method of diathermy coagulation for the primary lesion and teleradium to the groins, he has obtained a 60 per cent. five-year cure rate in a series of $8 \mathrm{I}$ patients without detectable lymph node metastases. Teleradium to the groins would, therefore, appear to be a method to recommend for these patients unless it is shown that prophylactic block dissection gives results sufficiently superior to those obtained by teleradium to counterbalance at least the mortality of the operation.

\section{Cancer of the Urethra}

It is difficult to make general statements concerning carcinoma of the urethra. The disease is so rare that no one person or centre has had sufficient opportunity to determine what is best for most patients. Carcinoma arising in the distal part of the urethra if seen early can be excised with preservation of the urethral sphincter or, as an alternative, radium implantation may be used. Each case must be judged on its own merits but as far as the local disease is concerned radiotherapy can be offered to the patient as a method equal in value to surgery.

The regional lymph node areas demand an active treatment policy. Inguinal lymph node involvement is frequent and often bilateral and the approach to treatment should be the same as for vulval cancer. Intrapelvic lymph node metastases are found in the more advanced cases, particularly when the posterior urethra is involved and these cases are rarely worth treating other than by palliative radiotherapy.

Various possible combinations of surgery and radiotherapy readily suggest themselves and find application according to individual circumstances and preference.

\section{Cancer of the Vagina}

Primary carcinoma of the vagina is also a very 
rare disease, although not quite so rare as primary carcinoma of the urethra. As with this latter disease it is impossible to give a generally applicable treatment policy owing to lack of opportunity of acquiring experience. The problem of treatment method, however, is simplified in the case of primary vaginal cancer since there is virtually no satisfactory surgical procedure available. Local excision cannot be regarded as adequate except for the very rare early lesion and more radical surgery is both excessively mutilating and does not give encouraging results. Radiotherapy must, therefore, be regarded as the treatment of choice for most cases.

The advanced case should be treated by external X-radiation supplemented, if possible, by local radium to the primary site; for the earlier cases, local radium treatment should be followed by X-ray therapy to the inguinal and intra-pelvic lymph node areas.

The local radium methods used depend on the part of the vagina affected. For the upper third of the vagina, the technique is the same as for the cervix uteri ; for the middle third, treatment by radium applicator should be used, and for the lower third, radium implantation. If the whole vaginal canal is invaded a central intracavitary source of radium should be employed.

\section{Acknowledgments}

I am deeply indebted to Sir Stanford Cade, K.B.E., F.R.C.S., for permission to draw upon material included in his book 'Malignant Disease and its Treatment by Radium.' 2nd Edition. Vol. III. Bristol: John Wright and Son, Ltd. (in press).

\section{BIBLIOGRAPHY}

RICHARDS, G. E. (1947), Proc. Roy. Soc. Med., 40, 912. GLUCKSMANN, A., and SPEAR, F. G. (1945), Brit. F. Radiol., 18, 3 I3.

GLUCKSMANN, A., and WAY, S. (1948), Ұ. Obst. and Gyn. Brit. Emp., 55, 573.

GRAHAM, R. (1947), Surg., Gyn. and Obst., 84, 166.

HULTBERG, S. (1944), Act. Radiol., 25, 59.

HEYMAN, J. (1943), Act. Obst. et Gyn. Scand., 23, 177.

ḦEYMAN, J. (1944), Act. Radiol., 25, 55 I.

TAUSSIG, F. J. (1943), Am. F. Obst. and Gyn., 45, 733.

HEYMAN, J., REUTERWALL, O., and BENNER, S. (194r), Act. Radiol., 22, 11.

HEYMAN, J. (1947), Brit. F. Radiol., $20,85$.

BERVEN, E. (194I), Act. Radiol., 22, 100.

\section{ROBERT LAWSON TAIT}

Robert Lawson Tait was born in Edinburgh in 1845 . His medical education under Simpson, Syme and Mathew Duncan resulted in his appointment as house surgeon after qualifying in 1866. He had enormous energy and courage which, with great technical ability, marked him out from his contemporaries. He set up practice in Birmingham and, by publishing his results, became known throughout Europe and America as the first founder of the principles of the surgery of the female pelvis, and the use of exploratory incisions in the abdomen. He used Syme's technique of scrupulous cleanliness without the use of boiled water, and can therefore not be credited with the introduction of aseptic technique. He died in 1895 with a reputation greater abroad than in this country because of the virulence with which he attacked Lister's principles and their protagonists. 